ANNALES

POLONICI MATHEMATICI

$83.2(2004)$

\title{
Lorentzian isothermic surfaces and Bonnet pairs
}

\author{
by M. A. Magid (Wellesley, MA)
}

\begin{abstract}
Lorentzian surfaces in Lorentz three-space are studied using an indefinite version of the quaternions. A classification theorem for Bonnet pairs in Lorentz three-space is obtained.
\end{abstract}

1. Introduction. In recent years there has been a resurgence of interest in certain classical differential geometric objects and constructions such as isothermic surfaces, Christoffel transforms and Darboux transforms. For example one has F. Burstall's epic work [B] and the wonderful example of both mathematics and scholarship [H-J]. These use, among other tools, Clifford algebras or quaternions to explore and clarify aspects of surface theory from the late 1800's. In [KPP] the quaternions are used to study isothermic surfaces and their relationship to what the authors define as Bonnet pairs.

In $[\mathrm{M}]$ it is shown that for Lorentzian isothermic surfaces, one has, just as in the positive definite setting, a dual surface. However, there are two types of isothermic surfaces and the constructions of the dual surfaces are different for the two types. One can still investigate these isothermic surfaces and appropriately defined Bonnet pairs in Lorentz space using what Libermann [L1, L2] called the quaternions of the second type $\widetilde{\mathbb{H}}$. These form a real, four-dimensional associative algebra containing some non-invertible, non-zero elements and give an appropriate setting for studying Lorentzian surfaces.

\section{Preliminaries}

2.1. Definitions and notations for Lorentzian surfaces in $\mathbb{R}_{1}^{3}$. The metric in $\mathbb{R}_{1}^{3}$ is denoted by

$$
\langle\vec{v}, \vec{w}\rangle=-v_{1} w_{1}+v_{2} w_{2}+v_{3} w_{3}
$$

2000 Mathematics Subject Classification: Primary 53A35.

Key words and phrases: Lorentzian surface, isothermic surface, quaternions.

I would like to thank Northeastern University for their hospitality during the writing of this paper and Franz Pedit for answering my many questions. 
for $\vec{v}=\left(v_{1}, v_{2}, v_{3}\right)$ and $\vec{w}=\left(w_{1}, w_{2}, w_{3}\right)$. We often use isothermal coordinates $(t, s)$ on our surface $M_{1}^{2}$ defined by an immersion $X: M_{1}^{2} \rightarrow \mathbb{R}_{1}^{3}$, by which we mean that, for the induced metric $h$ on $M_{1}^{2}$ and for the associated coordinate vectors $\partial t$ and $\partial s$, there is a non-zero function $\mu$ so that

$$
h(\partial t, \partial t)=-\mu^{2}, \quad h(\partial t, \partial s)=0, \quad h(\partial s, \partial s)=\mu^{2} .
$$

We can also use any set of isothermal coordinates to define null coordinates $\{x, y\}$ by

$$
x=\frac{t-s}{\sqrt{2}}, \quad y=\frac{t+s}{\sqrt{2}}
$$

so that $\partial x=\frac{1}{\sqrt{2}}(\partial t-\partial s), \partial y=\frac{1}{\sqrt{2}}(\partial t+\partial s)$ and $h(\partial x, \partial y)=-\mu^{2}$.

Following Weinstein [W, p. 13], a Lorentzian surface $\left(M_{1}^{2},[h]\right)$ is defined to be $M_{1}^{2}$ with all the metrics conformally equivalent to $h$.

The fundamental equations of the immersion $X$ are given by

$$
\begin{aligned}
X_{t t} & =\frac{\mu_{t}}{\mu} X_{t}+\frac{\mu_{s}}{\mu} X_{s}+e N, \\
X_{t s} & =\frac{\mu_{s}}{\mu} X_{t}+\frac{\mu_{t}}{\mu} X_{s}+f N, \\
X_{s s} & =\frac{\mu_{t}}{\mu} X_{t}+\frac{\mu_{s}}{\mu} X_{s}+g N, \\
N_{t} & =\frac{e}{\mu^{2}} X_{t}-\frac{f}{\mu^{2}} X_{s}, \\
N_{s} & =\frac{f}{\mu^{2}} X_{t}-\frac{g}{\mu^{2}} X_{s} .
\end{aligned}
$$

Thus, the shape operator corresponding to $N$ is

$$
A=\left[\begin{array}{cc}
-e / \mu^{2} & -f / \mu^{2} \\
f / \mu^{2} & g / \mu^{2}
\end{array}\right] .
$$

Recall that the shape operator at each point on a Lorentzian surface falls into one of three distinct classes which we call its algebraic type. The shape operator is either (a) diagonalizable over $\mathbb{R}$, (b) diagonalizable over $\mathbb{C}$ but not $\mathbb{R}$ or $(\mathrm{c})$ not diagonalizable over $\mathbb{C}$, and has a single real eigenvalue $\left(\left[\mathrm{O}^{\prime} \mathrm{N}\right]\right)$.

A Riemannian surface is called isothermic if there is an isothermal coordinate system for which every shape operator is diagonalized (over $\mathbb{R}$ ). For a Lorentzian surface we need to adjust the definition. Essentially we will call a surface isothermic if there is an isothermal coordinate system for which each shape operator is diagonalized over $\mathbb{R}$ or $\mathbb{C}$.

Definition 2.1. A Lorentzian immersion is called isothermic if there is some isothermal coordinate system $(t, s)$ such that each $A$ with respect to 
the basis $\{\partial t, \partial s\}$ has one of the following forms:
(a) $\left[\begin{array}{cc}\lambda_{1} & 0 \\ 0 & \lambda_{2}\end{array}\right]$,
(b) $\left[\begin{array}{cc}a & b \\ -b & a\end{array}\right]$.

We call the first case real isothermic and the second complex isothermic. In this paper we are working locally and define an isothermic immersion to be an immersion for which each point has a neighborhood which is either real isothermic or complex isothermic. Note that, in the complex isothermic case, the shape operator satisfies $e+g=0$.

Each Lorentzian surface $\left(M_{1}^{2},[h]\right)$ has a time orientation and an integrable product structure $J^{\prime}$. A time orientation is a choice of time cone in each tangent space. An integrable product structure is a tensor of type $(1,1)$ such that $J^{\prime 2}=\mathrm{Id}, J^{\prime} \neq \mathrm{Id}$ and $\nabla J^{\prime}=0$, where $\nabla$ is the Levi-Civita connection on $M_{1}^{2}$ defined by $h$. We define $J^{\prime}$ by choosing the two future directed null vectors $\{\partial x, \partial y\}$ in the chosen time cone so that $\{\partial x, \partial y\}$ is positively oriented, and set

$$
J^{\prime}(\partial x)=-\partial x, \quad J^{\prime}(\partial y)=\partial y .
$$

In the isothermal coordinates we have

$$
J^{\prime}(\partial t)=\partial s, \quad J^{\prime}(\partial s)=\partial t .
$$

The time orientation on $M_{1}^{2}$ is induced from the standard time orientation on $\mathbb{R}_{1}^{3}$; see [O'N, pp. 145 and 194]. We note that $J^{\prime}$ depends only on the Lorentzian surface $\left(M_{1}^{2},[h]\right)$, its time orientation, and orientation, which we include in the definition of $\left(M_{1}^{2},[h]\right)$. This structure is analogous to the complex structure defined on a Riemann surface.

2.2. Surfaces in $\widetilde{\mathbb{H}}$. Our $\mathbb{R}_{1}^{3}$ will sit in $\widetilde{\mathbb{H}}$, a four-dimensional Clifford algebra given by

$$
\widetilde{\mathbb{H}}=\left\{x_{0}+x_{1} i+x_{2} j^{\prime}+x_{3} k^{\prime} \mid x_{0}, x_{1}, x_{2}, x_{3} \in \mathbb{R}\right\}
$$

with

$$
\begin{gathered}
i^{2}=-1, \quad j^{2}=1=k^{\prime 2}, \\
i j^{\prime}=k^{\prime}, j^{\prime} i=-k^{\prime}, j^{\prime} k^{\prime}=-i, k^{\prime} j^{\prime}=i, i k^{\prime}=-j^{\prime}, k^{\prime} i=j^{\prime} .
\end{gathered}
$$

This was defined by Libermann in [L1], and called a quaternionic algebra of the second type. We will call them indefinite quaternions. For $x, y \in \widetilde{\mathbb{H}}$ we define our inner product $x \cdot y$ by

$$
x \cdot y=-\operatorname{Re}(\bar{x} y)=-x_{0} y_{0}-x_{1} y_{1}+x_{2} y_{2}+x_{3} y_{3},
$$

where $\overline{x_{0}+x_{1} i+x_{2} j^{\prime}+x_{3} k^{\prime}}=x_{0}-x_{1} i-x_{2} j^{\prime}-x_{3} k^{\prime}$. We call those indefinite quaternions with $x_{0}=0$ imaginary, and denote them by $\operatorname{Im} \widetilde{\mathbb{H}}$. They inherit a metric of signature $(1,2)$ from $\widetilde{\mathbb{H}}$ and are identified in all that follows with Lorentz three-space $\mathbb{R}_{1}^{3}$. The metric chosen here in $\widetilde{\mathbb{H}}$ has signature $(2,2)$ 
and induces the standard metric on the imaginary quaternions, but it is the negative of the one found in $[\mathrm{Po}]$. We denote $\widetilde{\mathbb{H}}$ by $\mathbb{R}_{2}^{4}$. Also note that if $x \in \operatorname{Im} \widetilde{\mathbb{H}}$ then $\bar{x}=-x$.

For imaginary $x$ and $y$ an important formula for calculations is

$$
x y=x \cdot y+x \times y,
$$

Here $x \times y$ is the cross product in $\mathbb{R}_{1}^{3}$ given by

$$
(x \times y) \cdot z=\operatorname{det}[z, x, y] .
$$

We can define, exactly as in $[\mathrm{KPP}]$, a wedge product on $\widetilde{\mathbb{H}}$-valued oneforms on $M_{1}^{2}$ by

$$
\alpha \wedge \beta(X, Y)=\alpha(X) \beta(Y)-\alpha(Y) \beta(X),
$$

which satisfies the identities

$$
\begin{aligned}
\overline{\alpha \wedge \beta} & =-\bar{\beta} \wedge \bar{\alpha}, & d(h \alpha) & =d h \wedge \alpha+h d \alpha, \\
\alpha \wedge h \beta & =\alpha h \wedge \beta, & d(\alpha h) & =d \alpha h-\alpha \wedge d h,
\end{aligned}
$$

where $h: M_{1}^{2} \rightarrow \widetilde{\mathbb{H}}$. We also identify two-forms on $M_{1}^{2}$ with their quadratic forms by

$$
\omega(X)=\omega\left(X, J^{\prime} X\right)
$$

for $\omega$ an $\widetilde{\mathbb{H}}$-valued two-form on $M_{1}^{2}$. If we define $* \alpha=\alpha \circ J^{\prime}$ then we have

$$
\alpha \wedge \beta=\alpha(* \beta)-(* \alpha) \beta,
$$

since $(\alpha \wedge \beta)\left(U, J^{\prime} U\right)=\alpha(U) \beta\left(J^{\prime} U\right)-\alpha\left(J^{\prime} U\right) \beta(U)$. Note that in the Lorentzian setting we have $*^{2}=\mathrm{Id}$. There is an algebraic lemma whose statement and proof is quite similar to one in [BFLPP].

Lemma 2.1. For $x, y \in \widetilde{\mathbb{H}}=\mathbb{R}_{2}^{4}$,

(1) $x y=y x$ iff $\operatorname{Im}(x)$ and $\operatorname{Im}(y)$ are linearly dependent over $\mathbb{R}$.

(2) $\operatorname{Im}\left(x^{2}\right)=0$ iff $x$ is either real or purely imaginary.

(3) $x^{2}=0$ iff $x \cdot x=0$, i.e., $x$ is in the null cone in $\mathbb{R}_{2}^{4}$.

(4) $x^{2}=1$ iff $x= \pm 1$ or $x$ is purely imaginary and $x^{2}=x \cdot x=1$.

(5) $x^{2}=-1$ iff $x$ is purely imaginary and $x^{2}=x \cdot x=-1$.

We also need the following version of Lemma 2.1 found in [KPP].

Lemma 2.2. $X: M_{1}^{2} \rightarrow \mathbb{R}_{1}^{3}$ is a conformal immersion iff there exists $N: M_{1}^{2} \rightarrow \widetilde{\mathbb{H}}$ such that

$$
(* d X) U=d X\left(J^{\prime} U\right)=N d X(U) .
$$

If (2.6) holds then $N: M_{1}^{2} \rightarrow S_{1}^{2} \subset \mathbb{R}_{1}^{3}$, where $S_{1}^{2}$ is the Lorentzian sphere in $\mathbb{R}_{1}^{3}$ and $N$ is the oriented unit normal field to $X$.

Proof. Assume first that $N$ exists. Since $d X$ is pointwise injective,

$$
d X(U)=(* d X)\left(J^{\prime} U\right)=(N d X)\left(J^{\prime} U\right)=N N d X(U),
$$


so that $N^{2}=1$. We see that $N$ cannot be \pm 1 , so that $N$ is purely imaginary and a map into the sphere. Next we would like to see that $N$ is normal to the surface. Note that $-\bar{N}=N$, so that $\overline{d X\left(J^{\prime} U\right)}=\overline{d X(U)} \bar{N}$ gives $-d X\left(J^{\prime} U\right)=d X(U) N$, hence $-N d X(U)=d X(U) N$ and

$$
N d X(U)+d X(U) N=0=2 N \cdot d X(U),
$$

so that $N$ is a normal field.

Conversely, suppose $X: M_{1}^{2} \rightarrow \mathbb{R}_{1}^{3}$ is a conformal immersion of a Lorentzian surface and assume that $N: M_{1}^{2} \rightarrow S_{1}^{2}$ is the unit normal field to $X$. We need to show that

$$
d X\left(J^{\prime} U\right)=N d X(U)=N \times d X(U) .
$$

Equivalently we must show that for the null coordinates $\{x, y\}$,

$$
d X(-\partial x)=N \times X_{x}, \quad d X(\partial y)=N \times X_{y} .
$$

We can see that this is so because $\operatorname{det}\left[X_{t} / \mu, X_{s} / \mu, N\right]=1$.

At this point we decompose our $\widetilde{\mathbb{H}}$-valued one-forms $\alpha=\alpha_{+}+\alpha_{-}$by defining

$$
\alpha_{+}=\frac{1}{2}(\alpha-N(* \alpha)), \quad \alpha_{-}=\frac{1}{2}(\alpha+N(* \alpha)) .
$$

These satisfy

$$
* \alpha_{+}=-N \alpha_{+}, \quad * \alpha_{-}=N \alpha_{-} .
$$

Now we can find

$$
\begin{aligned}
d(* d X) & =d(N d X)=d N \wedge d X=d N(* d X)-(* d N) d X \\
& =(d N N-* d N) d X=2\left(d N_{-}\right) N d X .
\end{aligned}
$$

We can see by a calculation involving the shape operator that $(d N N-* d N) d X$ is $\operatorname{Im} \widetilde{\mathbb{H}}$-valued. This says that $d N_{-} \cdot N d X=0$, which in turn implies that $d N_{-}$is a real multiple of $d X$. In fact we can see that $d N_{-}=H d X$, where $H$ is the negative of the mean curvature of the immersion $X$.

Thus we have

$$
d * d X=-2 H N d X \cdot d X
$$

and we can write

$$
d N=d N_{-}+d N_{+}=H d X+\theta \quad \text { with } \quad \theta=d N_{+} .
$$

We also get the Codazzi equation by differentiating (2.9):

$$
d \theta=(* d H-d H N) d X .
$$




\subsection{Spin equivalent immersions}

Definition 2.2. Two conformal immersions $X, \widetilde{X}:\left(M_{1}^{2},[h]\right) \rightarrow \mathbb{R}_{1}^{3}$ are called spin equivalent if there exists $\lambda: M_{1}^{2} \rightarrow \widetilde{\mathbb{H}}_{*}$ so that

$$
d \widetilde{X}=-\left(\frac{|\lambda \cdot \lambda|}{\lambda \cdot \lambda}\right) \bar{\lambda} d X \lambda=-\operatorname{sgn}(\lambda \cdot \lambda) \bar{\lambda} d X \lambda,
$$

where $\widetilde{\mathbb{H}}_{*}$ are the invertible elements.

The factor $\operatorname{sgn}(\lambda \cdot \lambda)$ is not needed in the positive definite case because it is always 1 there.

We note that, up to a positive scaling factor, this is an orthogonal transformation of $\mathbb{R}_{1}^{3}$ and the mapping

$$
\varrho(v)=-\frac{1}{\lambda \cdot \lambda} \bar{\lambda} v \lambda
$$

covers the special orthogonal group of $\mathbb{R}_{1}^{3}$. Note finally that the identity component of the special orthogonal group, which consists of orientation and time orientation preserving transformations, is generated by $\lambda$ with $\lambda \cdot \lambda<0$.

Thus we can see that, locally, any two conformal immersions are spin equivalent.

If $X$ and $\widetilde{X}$ are spin equivalent then we must have

$$
0=d(\bar{\lambda} d X \lambda)=d \bar{\lambda} \wedge d X \lambda-\bar{\lambda} d X \wedge d \lambda .
$$

This shows that $\bar{\lambda} d X \wedge d \lambda$ is real, so that $d X \wedge d \lambda=-\varrho d X \cdot d X \lambda=-\varrho d X d X \lambda$ for some real-valued function $\varrho$ on $M_{1}^{2}$. Then we have

$$
-\varrho d X d X \lambda=d X \wedge d \lambda=d X(* d \lambda)-(* d X) d \lambda=d X(* d \lambda+N d \lambda),
$$

so that we get the following integrability condition:

$$
-\varrho d X \lambda=* d \lambda+N d \lambda .
$$

We write this as a lemma:

Lemma 2.3. If $X$ and $\widetilde{X}$ are spin equivalent via

$$
d \tilde{X}=-\operatorname{sgn}(\lambda \cdot \lambda) \bar{\lambda} d X \lambda
$$

then $\lambda: M_{1}^{2} \rightarrow \widetilde{\mathbb{H}}_{*}$ satisfies

$$
-\varrho d X \lambda=* d \lambda+N d \lambda
$$

for some real-valued function $\varrho$ on $M_{1}^{2}$. Conversely, if $\pi_{1}\left(M_{1}^{2}\right)=0$ then for a given conformal immersion $X: M_{1}^{2} \rightarrow \mathbb{R}_{1}^{3}$, nowhere vanishing solutions $\lambda: M_{1}^{2} \rightarrow \widetilde{\mathbb{H}}_{*}$ to $(2.12)$ give all conformal immersions $\widetilde{X}: M_{1}^{2} \rightarrow \mathbb{R}_{1}^{3}$ via the transformations $d \widetilde{X}=-\operatorname{sgn}(\lambda \cdot \lambda) \bar{\lambda} d X \lambda$. 
The next lemma calculates the shape operator of a spin transform; it gives more information than the positive definite version.

Lemma 2.4. Let $X, \widetilde{X}: M_{1}^{2} \rightarrow \mathbb{R}_{1}^{3}$ be spin equivalent via $d \widetilde{X}=$ $-\operatorname{sgn}(\lambda \cdot \lambda) \bar{\lambda} d X \lambda$. Then

(1) $\widetilde{N}=\lambda^{-1} N \lambda$.

(2) $d \widetilde{X} \cdot d \widetilde{X}=(\lambda \cdot \lambda)^{2} d X \cdot d X$.

(3)

$$
\widetilde{A}=\frac{\operatorname{sgn}(\lambda \cdot \lambda)}{\lambda \cdot \lambda}\left[\begin{array}{cc}
-2 a_{2}-e / \mu^{2} & -f / \mu^{2}+2 a_{1} \\
-2 a_{1}+f / \mu^{2} & 2 a_{2}+2 \varrho+g / \mu^{2}
\end{array}\right],
$$

where $a_{1}, a_{2}$ are defined below.

Proof. The first two statements are clear so we will calculate the shape operator of $\widetilde{X}$. Using the fact that $N d \lambda=-* d \lambda-\varrho d X \lambda$ we find that

$$
N \lambda_{t}=-\lambda_{s}-\varrho X_{t} \lambda, \quad N \lambda_{s}=-\lambda_{t}-\varrho X_{s} \lambda,
$$

or

$$
\lambda_{t} \lambda^{-1}=-N \lambda_{s} \lambda^{-1}-\varrho X_{s}, \quad \lambda_{s} \lambda^{-1}=-N \lambda_{t} \lambda^{-1}-\varrho X_{t} .
$$

If we set

$$
\lambda_{t} \lambda^{-1}=a_{0}+a_{1} X_{t}+a_{2} X_{s}+a_{3} N, \quad \lambda_{s} \lambda^{-1}=b_{0}+b_{1} X_{t}+b_{2} X_{s}+b_{3} N,
$$

and use the equations above we see that

$$
b_{0}=-a_{3}, \quad b_{1}=-a_{2}-\varrho, \quad b_{2}=-a_{1}, \quad b_{3}=-a_{0} .
$$

We now calculate

$$
\begin{aligned}
d \widetilde{N} & =-\lambda^{-1} d \lambda \lambda^{-1} N \lambda+\lambda^{-1} d N \lambda+\lambda^{-1} N d \lambda \\
& =-\lambda^{-1}\left(d \lambda \lambda^{-1} N-d N-N d \lambda \lambda^{-1}\right) \lambda \\
& =\frac{1}{\lambda \cdot \lambda} \bar{\lambda}\left(d \lambda \lambda^{-1} N-d N-N d \lambda \lambda^{-1}\right) \lambda .
\end{aligned}
$$

Using the expression for $N_{t}$ and $N_{s}$ from (2.2) we can find

$$
\widetilde{A}=\frac{\operatorname{sgn}(\lambda \cdot \lambda)}{\lambda \cdot \lambda}\left[\begin{array}{cc}
-2 a_{2}-e / \mu^{2} & -f / \mu^{2}+2 a_{1} \\
-2 a_{1}+f / \mu^{2} & 2 a_{2}+2 \varrho+g / \mu^{2}
\end{array}\right] .
$$

Thus the negative of the mean curvature of $\widetilde{X}$ is

$$
\widetilde{H}=\frac{\operatorname{sgn}(\lambda \cdot \lambda)}{\lambda \cdot \lambda}(-\varrho+H)
$$

Corollary. Assume that $X, \tilde{X}: M_{1}^{2} \rightarrow \mathbb{R}_{1}^{3}$ are spin equivalent with

$$
d \tilde{X}=-\operatorname{sgn}(\lambda \cdot \lambda) \bar{\lambda} d X \lambda .
$$

Then the following are equivalent:

(1) $d X \wedge d \lambda=0$, which is the same as $* d \lambda+N d \lambda=0$.

(2) $\operatorname{sgn}(\lambda \cdot \lambda) \widetilde{H} d \widetilde{X} \cdot d \widetilde{X}=H(d X \cdot d X)(\lambda \cdot \lambda)$. 
3. Isothermic surfaces and Bonnet pairs. We begin with an alternative characterization of an isothermic surface.

Proposition 3.1. A surface $X: M_{1}^{2} \rightarrow \mathbb{R}_{1}^{3}$ is isothermic iff there is a two-dimensional closed one-form $\tau \in \Omega^{1}\left(M_{1}^{2}, \operatorname{Im} \widetilde{\mathbb{H}}\right)$ so that $* \tau+N \tau=0$, or, equivalently, $d X \wedge \tau=0$.

Proof. If a surface $X$ is real isothermic, then

$$
\tau=-\frac{X_{t}}{\mu^{2}} d t+\frac{X_{s}}{\mu^{2}} d s
$$

while if $X$ is complex isothermic,

$$
\tau=-\frac{X_{s}}{\mu^{2}} d t+\frac{X_{t}}{\mu^{2}} d s
$$

On the other hand, if such a locally integrable one-form $\tau$ is given, then there is a surface $X^{c}$ so that $d X \wedge d X^{c}=0$. Setting

$$
X_{t}^{c}=a_{1} X_{t}+b_{1} X_{s}+c_{1} N, \quad X_{s}^{c}=a_{2} X_{t}+b_{2} X_{s}+c_{2} N,
$$

and calculating $X_{t} X_{s}^{c}-X_{s} X_{t}^{c}=0$ gives

$$
X_{t}^{c}=a_{1} X_{t}+b_{1} X_{s}, \quad X_{s}^{c}=-b_{1} X_{t}-a_{1} X_{s} .
$$

We see that the tangent planes of the two surfaces agree. If $a_{1}^{2}-b_{1}^{2}>0$ the surface is real isothermic, while the other sign gives a complex isothermic surface $[\mathrm{M}]$. The only other possibility, $a_{1}^{2}-b_{1}^{2}=0$, does not yield a twodimensional one-form.

Definition 3.1. The Lorentzian isothermic surface $X^{c}$ in Proposition 3.1 is called the dual surface to $X$.

Definition 3.2. Two conformal immersions $X, \widetilde{X}:\left(M_{1}^{2},[h]\right) \rightarrow \mathbb{R}_{1}^{3}$ form a Bonnet pair if $d X \cdot d X=d \widetilde{X} \cdot d \widetilde{X}$, the principal curvatures agree, and the shape operators have the same algebraic type but they are not congruent. (If one avoids umbilic points then one only needs the mean curvatures to agree.)

Theorem 3.1. Let $X: M_{1}^{2} \rightarrow \mathbb{R}_{1}^{3}$ be an isothermic surface with dual $X^{c}: M_{1}^{2} \rightarrow \mathbb{R}_{1}^{3}$. Choose $\varepsilon \in \mathbb{R}, a \in \operatorname{Im} \widetilde{\mathbb{H}}$ and set $\lambda_{ \pm}= \pm \varepsilon+X^{c}+a$, where $\varepsilon$ and $a$ must be chosen so that $\lambda_{ \pm}$is invertible. Then the spin transforms $X_{ \pm}: M_{1}^{2} \rightarrow \mathbb{R}_{1}^{3}$ given by $d X_{ \pm}=-\operatorname{sgn}\left(\lambda_{ \pm} \cdot \lambda_{ \pm}\right) \bar{\lambda}_{ \pm} d X \lambda_{ \pm}$form a Bonnet pair.

Conversely, up to a rigid transformation, every pair of Bonnet surfaces arises from a three-parameter family (determined up to scalings) of isothermic surfaces where the three parameters account for the orientation and time orientation preserving rotations of the Bonnet pairs with respect to one another. 
Note that

$$
\begin{aligned}
\lambda_{ \pm} \cdot \lambda_{ \pm} & =-\left( \pm \varepsilon+X^{c}+a\right)\left( \pm \varepsilon-X^{c}-a\right) \\
& =-\left(\varepsilon^{2}-a^{2}-a X^{c}-X^{c} a-X^{c} X^{c}\right)=\left(X^{c}+a\right) \cdot\left(X^{c}+a\right)-\varepsilon^{2} .
\end{aligned}
$$

Thus the condition that $\lambda_{ \pm}$is invertible is equivalent to requiring that $X^{c}$ does not intersect the indefinite sphere centered at $-a$ with radius $\varepsilon$.

Proof. We first assume that we have $X$ and $X^{c}$ so that $d X \wedge d X^{c}=0$. This implies that $d X \wedge d \lambda_{ \pm}=0$ or $* d \lambda_{ \pm}+N d \lambda_{ \pm}=0$. This is (2.10) with $\varrho_{ \pm}=0$. We have seen that $\lambda_{+} \cdot \lambda_{+}=\lambda_{-} \cdot \lambda_{-}$, so that $d X_{+} \cdot d X_{+}=d X_{-} \cdot d X_{-}$

To see that $X_{ \pm}$form a Bonnet pair, we need to look at their shape operators. As we saw in the proof of Lemma 2.4 the shape operators depend on the shape operator of $X$ and the tangential components of $\left(\lambda_{+}\right)_{t}\left(\lambda_{+}\right)^{-1}$ and $\left(\lambda_{-}\right)_{t}\left(\lambda_{-}\right)^{-1}$. In fact these are

$$
\begin{aligned}
& \left(\lambda_{+}\right)_{t}\left(\lambda_{+}\right)^{-1}=\frac{1}{\lambda_{+} \cdot \lambda_{+}} X_{t}^{c}\left(-\varepsilon+X^{c}+a\right), \\
& \left(\lambda_{-}\right)_{t}\left(\lambda_{-}\right)^{-1}=\frac{1}{\lambda_{+} \cdot \lambda_{+}} X_{t}^{c}\left(\varepsilon+X^{c}+a\right) .
\end{aligned}
$$

We express the tangential component of these expressions respectively as

$$
\begin{aligned}
& \left(\left(\lambda_{+}\right)_{t}\left(\lambda_{+}\right)^{-1}\right)^{T}=\frac{1}{\lambda_{+} \cdot \lambda_{+}}\left(-\varepsilon X_{t}^{c}+c_{1} X_{t}+c_{2} X_{s}\right), \\
& \left(\left(\lambda_{-}\right)_{t}\left(\lambda_{-}\right)^{-1}\right)^{T}=\frac{1}{\lambda_{+} \cdot \lambda_{+}}\left(\varepsilon X_{t}^{c}+c_{1} X_{t}+c_{2} X_{s}\right) .
\end{aligned}
$$

We will now make a separate calculation for the two types of isothermic surfaces, real and complex. In the case of a real isothermic surface, $X_{t}^{c}=$ $-X_{t} / \mu^{2}$ and $c_{1}$ is zero. Indeed,

$$
\begin{aligned}
c_{1} & =\frac{1}{\mu^{2}} X_{t} \cdot\left(X_{t} / \mu^{2}\right)\left(X^{c}+a\right) \\
& =-\operatorname{Re} \frac{1}{\mu^{2}} \bar{X}_{t}\left(X_{t} / \mu^{2}\right)\left(X^{c}+a\right)=\frac{1}{\mu^{2}} \operatorname{Re}\left(X^{c}+a\right)=0 .
\end{aligned}
$$

Recalling that, for a real isothermic surface, $f=0$, we see that the shape operators of $X_{+}$and $X_{-}$are

$$
\begin{gathered}
A^{+}=\frac{\operatorname{sgn}\left(\lambda_{+} \cdot \lambda_{+}\right)}{\lambda_{+} \cdot \lambda_{+}}\left[\begin{array}{cc}
\frac{-2 c_{2}}{\lambda_{+} \cdot \lambda_{+}}-\frac{e}{\mu^{2}} & \frac{2 \varepsilon}{\left(\lambda_{+} \cdot \lambda_{+}\right) \mu^{2}} \\
\frac{-2 \varepsilon}{\left(\lambda_{+} \cdot \lambda_{+}\right) \mu^{2}} & \frac{2 c_{2}}{\lambda_{+} \cdot \lambda_{+}}+\frac{g}{\mu^{2}}
\end{array}\right], \\
A^{-}=\frac{\operatorname{sgn}\left(\lambda_{+} \cdot \lambda_{+}\right)}{\lambda_{+} \cdot \lambda_{+}}\left[\begin{array}{cc}
\frac{-2 c_{2}}{\lambda_{+} \cdot \lambda_{+}}-\frac{e}{\mu^{2}} & \frac{-2 \varepsilon}{\left(\lambda_{+} \cdot \lambda_{+}\right) \mu^{2}} \\
\frac{2 \varepsilon}{\left(\lambda_{+} \cdot \lambda_{+}\right) \mu^{2}} & \frac{2 c_{2}}{\lambda_{+} \cdot \lambda_{+}}+\frac{g}{\mu^{2}}
\end{array}\right] .
\end{gathered}
$$


Thus it is clear that, in this case, the shape operators have the same eigenvalues and the same algebraic type. Indeed, one can explicitly calculate the eigenvalues and eigenvectors to determine the algebraic type of the Bonnet pairs.

In the case of a complex isothermic surface, $X_{t}^{c}=-X_{s} / \mu^{2}$ and $c_{2}$ is zero as above. Recalling that, for a complex isothermic surface, $g=-e$ we see that the shape operators of $X_{+}$and $X_{-}$are

$$
\begin{gathered}
A^{+}=\frac{\operatorname{sgn}\left(\lambda_{+} \cdot \lambda_{+}\right)}{\lambda_{+} \cdot \lambda_{+}}\left[\begin{array}{cc}
\frac{-2 \varepsilon}{\mu^{2}\left(\lambda_{+} \cdot \lambda_{+}\right)}-\frac{e}{\mu^{2}} & \frac{2 c_{1}}{\lambda_{+} \cdot \lambda_{+}}-\frac{f}{\mu^{2}} \\
-\frac{2 c_{1}}{\lambda_{+} \cdot \lambda_{+}}+\frac{f}{\mu^{2}} & \frac{2 \varepsilon}{\mu^{2}\left(\lambda_{+} \cdot \lambda_{+}\right)}-\frac{e}{\mu^{2}}
\end{array}\right], \\
A^{-}=\frac{\operatorname{sgn}\left(\lambda_{+} \cdot \lambda_{+}\right)}{\lambda_{+} \cdot \lambda_{+}}\left[\begin{array}{cc}
\frac{2 \varepsilon}{\mu^{2}\left(\lambda_{+} \cdot \lambda_{+}\right)}-\frac{e}{\mu^{2}} & \frac{2 c_{1}}{\lambda_{+} \cdot \lambda_{+}}-\frac{f}{\mu^{2}}, \\
-\frac{2 c_{1}}{\lambda_{+} \cdot \lambda_{+}}+\frac{f}{\mu^{2}} & \frac{-2 \varepsilon}{\mu^{2}\left(\lambda_{+} \cdot \lambda_{+}\right)}-\frac{e}{\mu^{2}}
\end{array}\right] .
\end{gathered}
$$

Again, the shape operators have the same eigenvalues and the same algebraic type at each corresponding point.

For the converse suppose that $X_{-}$and $X_{+}$form a Bonnet pair and, initially, that $d X_{-}$and $d X_{+}$are never equal. We have

$$
d X_{+}=-\operatorname{sgn}(\lambda \cdot \lambda) \bar{\lambda} d X_{-} \lambda
$$

for some $\lambda: M_{1}^{2} \rightarrow \widetilde{\mathbb{H}}$. Since $X_{-}$and $X_{+}$induce the same metric, we have $d X_{+} \cdot d X_{+}=\bar{\lambda} d X_{-} \lambda \cdot \bar{\lambda} d X_{-} \lambda=(\bar{\lambda} \lambda)^{2} d X_{-} \cdot d X_{-}=d X_{-} \cdot d X_{-}$, so $\lambda \cdot \lambda= \pm 1$. We are assuming that $X_{ \pm}$induce the same time orientation, so that $\lambda \cdot \lambda=-1$. From equation (2.14) we see that $d X_{-} \wedge d \lambda=0$. We are looking for $\lambda_{ \pm}$so that

$$
d X_{ \pm}=-\operatorname{sgn}\left(\lambda_{ \pm} \cdot \lambda_{ \pm}\right) \bar{\lambda}_{ \pm} d X \lambda_{ \pm}
$$

with $\lambda_{+}=X^{c}+\varepsilon$ and $\lambda_{-}=X^{c}-\varepsilon$. In this case

$$
-\operatorname{sgn}\left(\lambda_{+} \cdot \lambda_{+}\right) \bar{\lambda}_{+} d X \lambda_{+}=-\operatorname{sgn}\left(\lambda_{-} \cdot \lambda_{-}\right) \overline{\lambda \lambda}_{-} d X \lambda_{-} \lambda .
$$

In order for this to hold we must have

$$
\lambda_{+}=\lambda_{-} \lambda .
$$

Thus, the signs of $\lambda_{-}$and $\lambda_{+}$are equal, yielding $\lambda_{-}+2 \varepsilon=\lambda_{+}$and $\lambda_{-}=$ $2 \varepsilon(\lambda-1)^{-1}$. We set $X^{c}=\varepsilon+2 \varepsilon(\lambda-1)^{-1}$. One can calculate, using $\lambda \cdot \lambda=-1$, that $X^{c}$ is imaginary.

Thus we can define $X$ by

$$
d X=-\operatorname{sgn}\left(\lambda_{-} \cdot \lambda_{-}\right) \frac{\bar{\lambda}-1}{2 \varepsilon} d X_{-} \frac{\lambda-1}{2 \varepsilon}
$$


since the forms on the right-hand side are closed. We can also see that $d X^{c} \wedge d X=0$.

We can now follow the proof of Theorem 2.3.2 in [KNPP]. Let $X_{ \pm}$be a Bonnet pair and $\lambda: M_{1}^{2} \rightarrow \mathbb{H}_{2}^{3}$ the function such that $d X_{+}=\bar{\lambda} d X_{-} \lambda$, where $\mathbb{H}_{2}^{3}$ is the set of vectors in $\widetilde{\mathbb{H}}$ of length -1 . Because $M_{1}^{2}$ is a surface, there must be an $a \in \mathbb{H}_{2}^{3}$ such that $a$ is not in the image of $\lambda$. We see that $-\operatorname{sgn}(a \cdot a) a X_{+} \bar{a}=a X_{+} \bar{a}$ and $X_{-}$form a Bonnet pair satisfying $d X_{-} \neq$ $a d X_{+} \bar{a}$.

\section{References}

[B] F. Burstall, Isothermic surfaces: conformal geometry, Clifford algebras and integrable systems, (October 11, 2000) arXiv:math.DG/0003096 v2.

[BFLPP] F. Burstall, D. Ferus, K Leschke, F. Pedit and U. Pinkall, Conformal Geometry of Surfaces in $S^{4}$ and Quaternions, Lecture Notes in Math. 1772, Springer, 2002 .

[H-J] U. Hertrich-Jeromin, Introduction to Möbius Geometry, London Math. Soc. Lecture Note Ser. 300, Cambridge Univ. Press, 2003.

[KNPP] G. Kamberov, P. Norman, F. Pedit and U. Pinkall, Quaternions, Spinors and Surfaces, Contemp. Math. 299, Amer. Math. Soc. Providence, RI, 2002.

[KPP] G. Kamberov, F. Pedit and U. Pinkall, Bonnet pairs and isothermic surfaces, Duke Math. J. 92 (1998), 637-644.

[L1] P. Libermann, Sur les structures presque quaternioniennes de deuxième espèce, C. R. Acad. Sci. Paris 234 (1952), 1030-1032.

[L2] - Sur les structures presque paracomplexes, ibid., 2517-2519.

[M] M. Magid, Lorentzian isothermic surfaces in $\mathbb{R}_{j}^{n}$, Rocky Mountain J. Math. to appear.

[O'N] B. O'Neill, Semi-Riemannian Geometry, Academic Press, New York, 1983.

[Po] I. Porteous, Clifford Algebras and the Classical Groups, Cambridge Univ. Press, Cambridge, 1995.

[W] T. Weinstein, Lorentz Surfaces, de Gruyter, Berlin, 1996.

Department of Mathematics

Wellesley College

Wellesley, MA 02481, U.S.A.

E-mail: mmagid@wellesley.edu 